\title{
ESTUDIO DE LA ORIENTACIÓN Y LA PERCEPCIÓN ESPACIAL EN ALUMNADO DEL GRADO DE EDUCACIÓN INFANTIL A TRAVÉS DE PORTAL 2
}

\author{
Juan Antonio Antequera Barroso \\ Departamento de Didáctica \\ Universidad de Cádiz \\ juanantonio.antequerar@uca.es \\ Jorge Guerra Antequera \\ Departamento Didáctica \\ Universidad de Extremadura \\ Ma Regina Teixeira Ferreira Capelo \\ CLEPUL/Polo of Madeira \\ John Miguel Costa Varela \\ CLEPUL/Polo of Madeira \\ Yésica del Águila Ríos \\ Departamento de Didáctica \\ Universidad de Cádiz
}

Fecha de Recepción: 24 Marzo 2019

Fecha de Admisión: 30 Abril 2019

\section{RESUMEN}

El videojuego es una herramienta cuya expansión y arraigo cultural en la sociedad actual permite su inclusión como elemento didáctico para trabajar con el alumnado.

El estudio se ha dirigido al alumnado del $2^{\circ}$ curso del grado de Educación Infantil de la Facultad de Ciencias de la Educación de la Universidad de Cádiz. Hemos empleado el videojuego Portal 2 para trabajar con nuestros estudiantes conceptos como la orientación y la percepción espacial. Conceptos de gran importancia en la etapa de Educación Infantil, etapa en la que los niños tienen su primer contacto con el colegio. De tal forma que los futuros maestros deberán aportar las herramientas suficientes a su futuro alumnado para relacionarse con el espacio que les rodea.

La metodología empleada en esta experiencia ha sido de carácter cuantitativo con un número total de estudiantes de 170. Se ha realizado un estudio exploratorio en 6 grupos. El procedimiento se ha llevado a cabo en tres fases diferenciadas: (a) Introducción sobre conceptos básicos del videojuego Portal 2. (b) Sesión de juego libre y guiado y (c) realización de un cuestionario.

Los resultados obtenidos, señalan que el alumnado adquiere ciertas destrezas relacionadas con la orientación y la percepción espacial y otros conocimientos relacionados con el campo matemático, tales como la toma de decisiones, tras el uso del videojuego Portal 2. 
Como conclusión, se puede señalar que el alumnado percibe al juego como un elemento de interés en el aula, aunque no necesariamente en el contexto educativo de la Educación infantil; sino que lo orientan a contextos educativos superiores como Educación Primaria y Secundaria. No obstante, consideran que su inclusión mejoraría la capacidad de abstracción, interpretación y percepción espacial.

Palabras clave: videojuego; orientación espacial; percepción espacial; educación infantil

\section{ABSTRACT}

Study of the guidance and spatial perception in pupils of the childhood education through portal 2. The video game is a tool whose expansion and cultural roots in today's society allows its inclusion as a didactic element to work with students.

The study has been directed to the students of the 2nd year of the Infant Education degree of the Faculty of Education Sciences of the University of Cadiz. We have used the video game Portal 2 to work with our students on concepts such as orientation and spatial perception. Concepts of great importance in the stage of Infant Education, stage in which the children have their first contact with the school. In such a way that the future teachers will have to contribute the sufficient tools to their future students to relate with the space that surrounds them.

The methodology used in this experience has been of a quantitative nature with a total number of students of 170. An exploratory study has been carried out in 6 groups. The procedure was carried out in three different phases: (a) Introduction to the basic concepts of the Portal 2 video game. (b) Free and guided play session and (c) completion of a questionnaire.

The results obtained indicate that the students acquire certain skills related to orientation and spatial perception and other knowledge related to the mathematical field, such as decision making, after the use of the video game Portal 2.

As a conclusion, it can be pointed out that students perceive play as an element of interest in the classroom, although not necessarily in the educational context of Early Childhood Education; rather, they orient it to higher educational contexts such as Primary and Secondary Education. Nevertheless, they consider that its inclusion would improve the capacity for abstraction, interpretation and spatial perception.

Keywords: videogame; spatial orientation; spatial perception; early childhood Education

\section{INTRODUCCIÓN}

La inclusión en de un videojuego en el ámbito educativo obedece a una tendencia al alza en la que destacan diversas corrientes pedagógicas como la Gamificación, en Edutainment o el Digital Game Based Learning (Prensky, 2001); siendo este último concepto en que se basará la implementación del videojuego Portal 2 en el aula de los futuros docentes de Educación Infantil de cara a conocer las posibilidades de dicho videojuego como herramienta educativa.

Diversos autores (Gros y Bernat, 2008; Guerra, 2017; López, 2014; McGonigal, 2010; Revuelta y Guerra, 2012) sostienen que los videojuegos son herramientas actuales que los discentes comprenden con facilidad. Son capaces de hacerse con los controles, la interfaz y las mecánicas en muy poco tiempo debido a su uso cotidiano. Por lo tanto, el docente tan sólo debería readaptar el leit motiv del videojuego en base a sus pretensiones pedagógicas. En el caso de este experimento el videojuego se desarrolla bajo una a priori compleja trama, que si bien es cierto que incluye múltiples conceptos es capaz de sintetizarlos y verterlos al jugador de un modo sencillo e intuitivo con una interpretación relativamente asequible para los profanos de los campos anteriormente detallados. 


\section{EL ÁMBITO ESPACIAL-GEOMÉTRICO EN EDUCACIÓN INFANTIL}

A lo largo de nuestra vida para poder desplazarnos por los lugares que nos rodean planteamos una serie de puntos de referencia que son los que nos favorecen en el movimiento, por ejemplo, paseando por una nueva ciudad nos fijamos en los comercios, bares o monumentos que están en el camino a nuestro hotel o desde el mismo. Todos estos mecanismos para orientarnos y estructurar el espacio que nos rodea se van desarrollando a lo largo de toda nuestra vida, teniendo especial importancia en las primeras edades. En concreto, en el periodo de Educación Infantil, de 0 y 6 años.

Durante la etapa de Educación Infantil, se adquieren una serie de conocimientos que van relacionados con su propio cuerpo, así como con el entorno que le rodea. De tal forma que puede establecer relaciones entre su propio cuerpo, los objetos y el espacio circundante a través de la exploración. Todo esto de acuerdo con la teoría de Piaget e Inhelder (1948, citado por Alsina 2011) distinguiéndose dos periodos: el periodo sensoriomotor (de los 0 a los 2 años aproximadamente) y el periodo representacional (de los 2 a los 12 años)

El niño empleando como sistema de referencia su propio cuerpo establece las primeras relaciones con el entorno que le rodea. A partir de sí mismo es capaz de observar los objetos o personas que tiene delante 0 detrás. De igual forma, aquellos que están a su izquierda 0 derecha 0 encima 0 debajo, por ejemplo, de una mesa. También aquellos que están próximos o separados de él, por ejemplo, cuando es bebé de su madre. Así como, es capaz de distinguir conceptos como abierto 0 cerrado (interior y exterior) en referencia por ejemplo a su propio cuarto. Todos estos conceptos están ligados con relaciones de tipo topológico.

Las siguientes relaciones que el niño es capaz de trabajar son de tipo proyectivo. En este caso entra en juego la perspectiva del niño a la hora de observar los objetos. El niño puede comprobar cómo cambian los objetos según el lugar desde el que se están observando. Por ejemplo, si se observa un objeto colocado sobre una mesa depende donde estemos sentado lo veremos de una forma u otra. Pero en conjunto tendremos el objeto completo.

Y, por último, el niño ya es capaz de desplazarse por el espacio y realizar operaciones como giros, simetrías o traslaciones. Así como, distancias, tamaños y direcciones de los objetos o personas que se encuentran en el espacio circundante. A este tipo de relaciones se denominan euclídeas 0 métricas.

\section{Videojuegos y el ámbito Geométrico-Espacial}

A la hora de trabajar las relaciones descritas anteriormente los videojuegos se muestran como una herramienta didáctica de gran valor. En concreto, los videojuegos permiten a la persona que los están utilizando desarrollar las relaciones topológicas, proyectivas y métricas 0 euclídeas de una manera sencilla y, a veces, de manera casi inconsciente a la hora de resolver el problema que plantea el nivel del juego.

Lo primero que hace la persona que se enfrenta al juego es observar el espacio que le rodea, realizando así una orientación y estructuración del mismo. A continuación, decide la estrategia o táctica a seguir para resolver la pantalla o nivel en el que se está jugando. Todo esto conlleva un alto grado de abstracción (McGonigal, 2010) que es propio de una disciplina como las Matemáticas.

Con respecto a las relaciones cabe destacar el uso de las topológicas al decidir los movimientos que se van a realizar en función de la disposición de los objetos que aparecen en la pantalla. Por ejemplo, si el personaje se encuentra en una sala cerrada de la que tiene que salir trabajaría conceptos topológicos de recinto o cerramiento, proximidad, etc. De igual forma aparecen las proyectivas ya que se deberá prever qué es lo que puede encontrarse detrás de una estructura en función de lo que está observando en el momento, es decir, del punto de vista del jugador. Y, por último, las 
de tipo de métrico. En este caso, por ejemplo, entra en juego la decisión del jugador a la hora de realizar un salto entre plataformas evaluando así la distancia entre plataformas o bloques.

De ahí, que los videojuegos se manifiesten como una herramienta didáctica que permite desarrollar 0 analizar una serie de conceptos abstractos de una manera lúdica y cercana.

\section{MÉTODO}

\section{Objetivo}

El objetivo sobre el que se plantea esta investigación es conocer la percepción del alumnado del Grado de Educación Infantil de la Facultad de Educación de la Universidad de Cádiz sobre la utilización del videojuego Portal 2 para trabajar nociones matemáticas sobre la orientación y la percepción espacial.

\section{Población y Muestra}

La población está constituida por alumnas y alumnos de la Universidad de Cádiz. La muestra está conformada por 170 individuos ( $n=170$ ) que cursan segundo curso del Grado de Educación Infantil. La creciente incorporación de los videojuegos a contextos formativos hace que sea necesario conocer su percepción y posibilidades educativas como futuros docentes, de ahí la selección de esta muestra.

A continuación, se presentan una serie de tablas y gráficos relativos a los datos sociodemográficos de la muestra.

Tabla 1.

Datos referentes al sexo de los individuos participantes del estudio. Fuente: elaboración propia

\begin{tabular}{lcc}
\hline & Frecuencia & Porcentaje \\
\hline Mujer & 161 & $95 \%$ \\
\hline Hombre & 9 & $5 \%$ \\
\hline Total & 170 & 100,0 \\
\hline
\end{tabular}

Gráfico 1. Porcentaje de individuos separados por sexos. Fuente: elaboración propia.

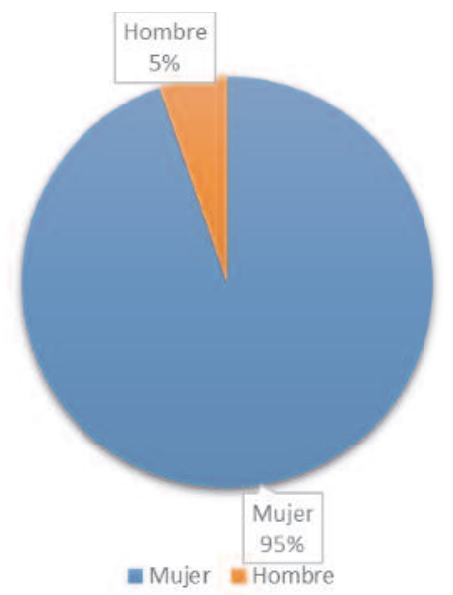


Encontramos un número de mujeres significativamente elevado respecto a los hombres.

Tabla 2 .

Datos referentes a la edad de los individuos participantes del estudio. Fuente: elaboración propia

\begin{tabular}{lcc}
\hline & Frecuencia & Porcentaje \\
\hline 18 años & 2 & $1 \%$ \\
\hline 19 años & 56 & $33 \%$ \\
\hline 20 años & 17 & $10 \%$ \\
\hline 21 años & 26 & $15 \%$ \\
\hline 23 años & 26 & $15 \%$ \\
\hline 24 años & 22 & $13 \%$ \\
\hline 25 años & 13 & $8 \%$ \\
\hline 26 años & 4 & $2 \%$ \\
\hline 50 años & 1 & $1 \%$ \\
\hline
\end{tabular}

Los individuos que componen la muestra se distribuyen principalmente en el rango de edad de 18 a 24 años (Tabla 2). No obstante, puede observarse que existe diversidad entre las edades de los individuos estudiados.

\section{Procedimiento}

Se ha realizado un estudio exploratorio en la muestra seleccionada. Que ha constado de tres fases. (a) Introducción sobre conceptos básicos relativos a los controles y la narrativa del videojuego Portal 2. (b) Sesión de juego libre para la asimilación de los controles seguido de juego guiado en escenarios preestablecidos con obstáculos y laberintos relacionados con la percepción espacial. (c) Realización de un cuestionario sobre la idoneidad de Portal 2 como recurso significativo en Educación Infantil para trabajar la percepción espacial.

\section{Instrumento}

Los instrumentos utilizados en este estudio han sido dos: (a) un cuestionario (Fig. 1) con preguntas relativas a las posibilidades educativas a Portal 2 respecto a la percepción espacial, la geometría 0 la lógica creado con Google Drive. Y el (b) videojuego desarrollado por Valve Portal 2 (Fig. 2).

Figura 1. Captura de pantalla del cuestionario. Fuente: elaboración propia.

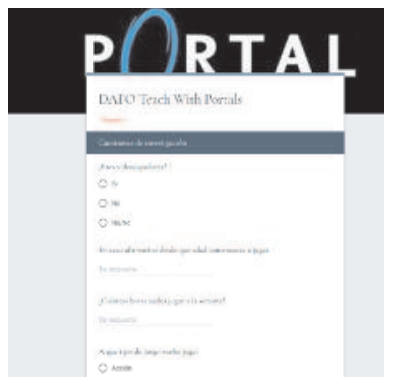


Figura 2. Captura de pantalla de Portal 2. Fuente: elaboración propia.

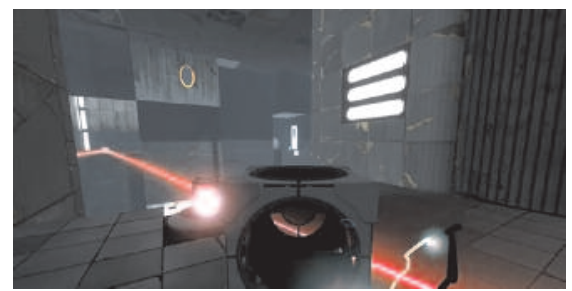

Técnicas de análisis

Para el análisis estadístico se ha utilizado el sistema de análisis estadístico Statistical Package for the Social Sciences (SPSS) en su versión 24.

\section{RESULTADOS}

Los hallazgos que se han presentado en esta investigación muestran que el alumnado considera que Portal 2 puede ser un elemento a considerar en Educación Infantil para trabajar la percepción y la orientación espacial. A continuación, se expondrán los datos recabados en diferentes tablas.

\section{Tabla 3.}

Datos referentes a la cuestión ¿eres videojugador/a? Fuente: elaboración propia

\begin{tabular}{ccc}
\hline & Frecuencia & Porcentaje \\
\hline $\mathrm{Si}$ & 46 & $27 \%$ \\
\hline $\mathrm{No}$ & 124 & $73 \%$ \\
\hline
\end{tabular}

De los 170 individuos que componen la muestra 46 declaran ser videojugadores/as, lo cual representa un $27 \%$. Los individuos que no se consideran videojugadores/as son 124 ; siendo el $73 \%$ del total. Atendiendo a las respuestas dadas y los resultados obtenidos, se consideran videojugadores aquellos individuos que poseen una rutina de juego. Los no jugadores son aquellos que o no juegan habitualmente 0 juegan rara vez. Dado el amplio número de mujeres, se ha decidido desgranar la pregunta anterior para realizar una separación por sexos que ayude a precisar sobre los datos obtenidos.

Tabla 4.

Datos referentes a la cuestión ¿eres videojugador/a? para mujeres. Fuente: elaboración propia

\begin{tabular}{ccccc}
\hline & \multicolumn{2}{c}{ Mujer } & \multicolumn{2}{c}{ Hombre } \\
\hline & Frecuencia & Porcentaje & Frecuencia & Porcentaje \\
\hline $\mathrm{Si}$ & 41 & $26 \%$ & 5 & $56 \%$ \\
\hline $\mathrm{No}$ & 114 & $71 \%$ & 4 & $44 \%$ \\
\hline Ns/Nc & 6 & $3 \%$ & 0 & $0 \%$ \\
\hline Total & 161 & & 9 & \\
\hline
\end{tabular}

Puede apreciarse un significativo número de mujeres respecto del total de la muestra, 161. No obstante, tan solo 41 , el $26 \%$ del total son videojugadoras y 114 no se consideran habituales. Finalmente, 6 no se identifican con ninguno de los dos perfiles. En el caso de los hombres, 5 de ellos, aseguran ser videojugadores habituales y los 4 restantes no lo hace. 
Una vez conocido, el perfil en el que los individuos analizados creen encontrarse. Se les preguntó ¿cuántas horas sueles jugar a la semana? A continuación, pueden verse los resultados obtenidos (Tabla 5).

Tabla 5.

Frecuencia de uso semanal de videojuegos (horas). Fuente: elaboración propia

\begin{tabular}{ccc}
\hline (horas) & Frecuencia & Porcentaje \\
\hline 1 & 9 & $5 \%$ \\
\hline 2 & 10 & $6 \%$ \\
\hline 3 & 8 & $5 \%$ \\
\hline 4 & 3 & $2 \%$ \\
\hline 5 & 4 & $2 \%$ \\
\hline 7 & 1 & $1 \%$ \\
\hline 12 & 2 & $1 \%$ \\
\hline 14 & 1 & $1 \%$ \\
\hline 15 & 1 & $1 \%$ \\
\hline 20 & 1 & $1 \%$ \\
\hline 27 & 1 & $1 \%$ \\
\hline 30 & 1 & $1 \%$ \\
\hline 50 & 1 & $1 \%$ \\
\hline Total & 50 & $30 \%$ \\
\hline & &
\end{tabular}

Las respuestas obtenidas han sido 50 de 170; representando el 30\% del total. El $70 \%$ restante no respondió debido a que no se han declarado videojugadores. De los que respondieron afirmativamente, podemos observar los valores más habituales están entre 1 hora a 5 horas; siendo $M=$ 5.44 y dt=9.121. Existiendo valores de uso inusual como pueden ser 30050 horas.

Tabla 6.

Género de videojuegos utilizados por los individuos analizados. Fuente: elaboración propia

\begin{tabular}{ccc}
\hline & Frecuencia & Porcentaje \\
\hline Aventuras & 18 & $11 \%$ \\
\hline Estrategia & 19 & $11 \%$ \\
\hline Acción & 10 & $6 \%$ \\
\hline Simulación & 16 & $9 \%$ \\
\hline Deportivo & 8 & $5 \%$ \\
\hline Casual & 5 & $3 \%$ \\
\hline Total & 76 & $45 \%$ \\
\hline
\end{tabular}

Se les cuestionó sobre su género de videojuego favorito, ¿a qué tipo de juego sueles jugar? (Tabla 6). Respondieron 76 individuos, un 45\% del total. Los géneros favoritos fueron el de aventuras y estrategia con un $11 \%$ y simulación con un 10\%. Cabe señalar que son géneros primarios de videojuegos presentes en todas las plataformas.

Tabla 7.

Resultados relativos a la razón del uso de videojuegos. Fuente: elaboración propia

\begin{tabular}{lcc}
\hline & Frecuencia & Porcentaje \\
\hline Para pasar el tiempo con los amigos & 7 & $4 \%$ \\
\hline Por diversión & 48 & $28 \%$ \\
\hline Para relajarme & 9 & $5 \%$ \\
\hline Para evadirme & 7 & $4 \%$ \\
\hline Para aprender & 3 & $2 \%$ \\
\hline Total & 74 & $44 \%$ \\
\hline
\end{tabular}


Respecto a los resultados arrojados para la pregunta ¿Por qué juegas a videojuegos? (Tabla 7). Fue respondida por 74 individuos, el 44\%. Muestra que la mayor parte de los individuos, 48, que respondieron la encuesta los utilizan como medio de diversión, es decir, vinculan su uso a la consideración lúdica. En este sentido, también pueden considerarse a los individuos que los utilizan como instrumento de relajación, un 5\%. En el caso del uso por evasión e instrumento social para pasar el tiempo con los amigos, 7 han sido los que han considerado esta respuesta; representando en ambos casos el 4\%. Finalmente, tan sólo 3, el 2\% los utilizan con algún tipo de pretensión educativa.

Tabla 8.

Resultados relativos a la consideración educativa de los videojuegos. Fuente: elaboración propia

\begin{tabular}{lcc}
\hline & Frecuencia & Porcentaje \\
\hline Nada & 26 & $15 \%$ \\
\hline Poco & 10 & $6 \%$ \\
\hline Algo & 65 & $38 \%$ \\
\hline Bastante & 7 & $4 \%$ \\
\hline Mucho & 62 & $37 \%$ \\
\hline
\end{tabular}

Para la cuestión ¿crees que los videojuegos son educativos? (Tabla 8). La mayor parte de las respuestas, el 38\% consideran que los videojuegos pueden ser educativos moderadamente. 62 de los individuos analizados consideran que los videojuegos son recursos de alta significatividad educativa; representando el 37\%. Otro grupo, el 4\%, mantiene que estos recursos son moderadamente significativos. Consideran que los videojuegos son recursos con nulo interés educativo el $15 \%$ del total. Otros, $6 \%$ del total, mantienen que los videojuegos son poco relevantes en cuanto a su adhesión educativa.

Tabla 9.

Resultados relativos a las respuestas obtenidas para el fundamento de la utilización o no de los videojuegos en el aula. Fuente: elaboración propia

\begin{tabular}{lcc} 
& Frecuencia & Porcentaje \\
\hline Generan violencia y adicción & 31 & $18 \%$ \\
\hline Generan aprendizajes & 71 & $42 \%$ \\
\hline Generan creatividad & 1 & $1 \%$ \\
\hline Su fin es lúdico & 29 & $17 \%$ \\
\hline Los videojuegos no tienen utilidad & 19 & $11 \%$ \\
\hline Total & 151 & $89 \%$ \\
\hline
\end{tabular}

A los individuos analizados se les preguntó sobre la posibilidad de justificar la respuesta anterior en la que se hacía alusión al agrado de adhesión educativa de los videojuegos (Tabla 9). Analizadas sus respuestas se han establecido cinco categorías en las que se han ubicado todas las respuestas. Del total de 170 individuos, 151 respondieron a esta pregunta; siendo el $89 \%$. Atendiendo a los datos recabados, el $42 \%$ consideran que los videojuegos generan algún tipo de aprendizaje. En la contraparte, existen 31 individuos que consideran que los videojuegos generan algún tipo de disfuncionalidad en el comportamiento manifestándose en actitudes violentas 0 adictogénicas. El 17\% cree que los videojuegos son recursos plenamente lúdicos sin pretensiones. Un individuo, manifiesta la potencialidad alrededor de la creatividad. Finalmente, 19 individuos; el 11\%, mantienen que los videojuegos carecen de utilidad alguna. 
Tabla 10.

Resultados relativos a las respuestas obtenidas para el fundamento de la utilización o no de los videojuegos en el aula. Fuente: elaboración propia

\begin{tabular}{lcc}
\hline & Frecuencia & Porcentaje \\
\hline $\mathrm{Si}$ & 151 & $89 \%$ \\
\hline No & 19 & $11 \%$ \\
\hline
\end{tabular}

Tras conocer su opinión sobre la vinculación educativa de los videojuegos. Se preguntó ¿crees que Portal 2 podría ser aplicado al aula? Para conocer si el alumnado consideraba que podía ser una herramienta capaz de ser introducida en contextos formales de aprendizaje. Los datos señalan que 151 de los individuos analizados, 89\% consideran que pueden ser utilizados de algún modo y 19 de éstos; representando el $11 \%$ no lo creen viable.

\section{Tabla 11.}

Resultados relativos a las respuestas obtenidas la consideración de viabilidad de los videojuegos según el contexto educativo. Fuente: elaboración propia

\begin{tabular}{lcc}
\hline & Frecuencia & Porcentaje \\
\hline Infantil & 8 & $5 \%$ \\
\hline Primaria & 79 & $46 \%$ \\
\hline Secundaria & 64 & $38 \%$ \\
\hline Universidad & 12 & $7 \%$ \\
\hline Formación profesional & 2 & $1 \%$ \\
\hline Contextos informales de aprendizaje & 5 & $3 \%$ \\
\hline
\end{tabular}

La vinculación con el ámbito educativo necesitaba precisarse por lo que se les emplazó a responder a la cuestión ¿en qué niveles sería más viable? (Tabla 11). La mayor parte de los individuos, el $46 \%$ cree que son más afines para ser utilizados en Educación Primaria. Otro de los grupos significativos es el 38\% que considera que son idóneos para ser utilizados en Educación Secundaria. Respecto a la utilización de los videojuegos en la Universidad, 12 individuos, el 7\% creen son recursos de utilidad en este nivel formativo. En Educación Infantil, el 5\% sostiene que en este nivel educativo pueden introducirse videojuegos como recurso. Respecto a los contextos informales de aprendizaje, alejados de instituciones y con un marcado carácter espontáneo, son considerados por el 3\%. Finalmente, un 1\% mantiene que son propicios para ser utilizados en Formación Profesional. Se puede observar, que el alumnado no considera suficientemente consistente la idea de implementar videojuegos en el aula de Infantil respecto a otros niveles educativos.

Tabla 12.

Resultados relativos a la asimilación de los controles del juego por parte del alumnado de Educación Infantil. Fuente: elaboración propia

\begin{tabular}{lcc}
\hline & Frecuencia & Porcentaje \\
\hline $\mathrm{Si}$ & 120 & $71 \%$ \\
\hline $\mathrm{No}$ & 41 & $24 \%$ \\
\hline $\mathrm{Ns} / \mathrm{Nc}$ & 9 & $5 \%$ \\
\hline
\end{tabular}


En relación a la cuestión planteada ¿crees que los discentes podrían hacerse con el control fácilmente? se pregunta a los individuos investigados sobre si consideran que los controles del videojuego Portal son asumibles para un niño o niña cuya edad está comprendida en la etapa de Educación Infantil. Respondieron afirmativamente el $71 \%$, negativamente el $24 \%$ y el $5 \%$ restante, no fueron capaces de posicionarse en ninguna de las dos opciones propuestas.

Tabla 13.

Resultados relativos a la comprensión de la narrativa que vertebra el juego Portal 2 para el alumnado de Educación infantil. Fuente: elaboración propia

\begin{tabular}{lcc}
\hline & Frecuencia & Porcentaje \\
\hline $\mathrm{Si}$ & 118 & $69 \%$ \\
\hline $\mathrm{No}$ & 45 & $27 \%$ \\
\hline $\mathrm{Ns} / \mathrm{Nc}$ & 7 & $4 \%$ \\
\hline
\end{tabular}

La narrativa sobre la que se estructura el juego y su posible comprensión por el alumnado de Educación Infantil fue cuestionada a los futuros docentes con la pregunta ¿crees que comprenderían el sentido del juego? Si bien es cierto que dado que la temática es muy compleja para su edad: agujeros de gusano, física de portales, líquidos no Newtonianos, etc. La estructura del juego se desarrolla en un plano lineal o capsular (habitaciones) con los accesorios necesarios para salir de ellas. El alumnado en su mayoría el 69\% considera que los discentes de Educación Infantil podrían entender las mecánicas dada la simplicidad de la propuesta y los controles sencillos. Sin embargo, 45 el $27 \%$ considera que debido a la densidad del argumento de los niveles principales no es una herramienta apropiada para ser incluida en las aulas de Educación infantil. El 4\% restante, no fue capaz de determinar su posición respecto a esta cuestión.

Tabla 14.

Resultados relativos la adhesión del videojuego Portal 2 a las áreas de Educación Infantil. Fuente: elaboración propia.

\begin{tabular}{lcc}
\hline & Frecuencia & Porcentaje \\
\hline Conocimiento del entorno & 130 & $76 \%$ \\
\hline Conocimiento de sí mismo y autonomía personal. & 30 & $18 \%$ \\
\hline Lenguaje. Comunicación y representación & 10 & $6 \%$ \\
\hline
\end{tabular}

Tras probar el juego era importante la perspectiva de los futuros docentes de Educación Infantil sobre la posible implicación del videojuego Portal 2 en el aula. Y dado el carácter multidisciplinar y heterogéneo de los videojuegos se les ha cuestionado sobre las qué áreas de las que se trabajan en Educación Infantil son más proclives al trabajo con dicho videojuego. La mayor parte de los individuos analizados, el $76 \%$ del total, considera que es afín al área Conocimiento del Entorno. Dada las posibilidades de interacción de los elementos presentes en el juego con el jugador, otros NPC (Non Playable Characters - personajes no jugables), otros elementos o el propio entorno. Es por ello, que esta opción parece ser la idónea según su criterio. El conocimiento de sí mismo y autonomía personal fue seleccionada por el 18\%. Los constante requerimientos de superación de obstáculos y desafíos pone a prueba a los individuos y les alienta a ser resolutivos con recursos escasos; supe- 
rando la frustración y generando iniciativa ante los retos. En el caso del área de Lenguaje. Comunicación y representación, los individuos que se decantaron por ella el 6\%. Dadas las múltiples órdenes, señales, iconos y gráficos existentes en el juego podrían ser utilizados como mensajes de ayuda o complementos para la resolución de los obstáculos principales, ayudando a comprender como funcionan los diferentes sistemas comunicativos.

Tabla 15.

Resultados relativos al modo de introducir Portal 2 en el aula de Educación Infantil. Fuente: elaboración propia.

\begin{tabular}{lcc}
\hline & Frecuencia & Porcentaje \\
\hline Recurso principal & 8 & $5 \%$ \\
\hline Recurso complementario & 99 & $58 \%$ \\
\hline Anecdótico (como motivación lúdica) & 61 & $36 \%$ \\
\hline Recurso que carece de utilidad en el aula & 2 & $1 \%$ \\
\hline
\end{tabular}

Conociendo en qué área ubicarían al videojuego Portal 2 se cuestionó a los discentes sobre qué consideración le darían al videojuego en el aula con la cuestión ¿cómo introducirías Portal 2 en el aula? La mayor parte de los individuos investigados, el 58\%, considera que el videojuego Portal 2 debe ser utilizado como un recurso complementario a los recursos habituales del aula. En este sentido, el $36 \%$ considera que su uso debe limitarse a lo anecdótico, sin unas pretensiones educativoformativas más allá de alguna sesión extraordinaria para mostrar un recurso curioso y/o interesante. La consideración como recurso principal vertebrador de aprendizajes es mantenida por el $5 \%$ del total. Respecto a la consideración de Portal 2 como elemento carente de utilidad en el aula, es mantenida por el 1\%. Se señala que un videojuego es lúdico y como tal su inclusión en el aula se realiza desde esta perspectiva.

Cabe señalar una tónica general en todas las respuestas es la falta de formación docente para la implementación de estas herramientas en el aula. Conocen la existencia de los recursos, pero carecen de conocimientos y medios para abordarlos desde una perspectiva didáctica.

Tabla 16.

Resultados relativos al modo de aplicar Portal 2 en el aula de Educación Infantil para el conocimiento matemático. Fuente: elaboración propia.

\begin{tabular}{lcc}
\hline & Frecuencia & Porcentaje \\
\hline Conocimiento viso-espacial & 74 & $44 \%$ \\
\hline Conocimiento lógico-matemático & 54 & $32 \%$ \\
\hline Conocimiento geométrico & 20 & $12 \%$ \\
\hline Lateralidad & 6 & $3 \%$ \\
\hline Conteo & 2 & $1 \%$ \\
\hline Autonomía personal & 11 & $6 \%$ \\
\hline Ns/Nc & 3 & $2 \%$ \\
\hline
\end{tabular}

Precisando sobre la adhesión del videojuego Portal 2 al aula de Educación Infantil y focalizando en la asignatura de matemáticas, contexto en el que la investigación fue realizada. Se traslada los individuos analizados la siguiente cuestión ¿dentro de la asignatura de matemáticas destaca qué aprendizajes se podrían tratar con Portal 2? El conocimiento viso-espacial fue el contenido por el que se decantaron el mayor porcentaje de los discentes, el 44\%. La elección de éste obedece a la mecánica del juego que permite interaccionar con el espacio de juego desde diferentes perspectivas 
además del añadido de la mecánica de que aporta la física de portales; permitiendo a los videojugadores/as experimentar el espacio de interacción de diversos modos.

En sintonía con la asignatura de conocimiento lógico-matemático fue seleccionado por un $32 \%$. Esta elección se debe a las mecánicas de juego en formato de resolución de enigmas y puzles, por lo que el individuo ha de vincular diferentes elementos y mecánicas para resolver enigmas que posibiliten avanzar en el juego.

En alusión al conocimiento geométrico, el 12\% sostiene que el videojuego les ayuda a comprender el concepto relativo a las dimensiones y su lugar en el espacio debido a las posibilidades de desplazamiento 3D y las diferentes perspectivas.

Otro de los contenidos que los individuos investigados han destacado ha sido la autonomía personal, el $6 \%$, sostiene que el videojuego les emplaza ante un reto constante al que deben dar solución con los elementos presentes en cada cámara o nivel. Salir de ahí depende de su destreza deduciendo e interaccionando con dichos elementos.

El trabajo con la lateralidad es seleccionado por el 3\%. Ello es debido a que han focalizando más en el manejo del recurso que en el propio recurso. Señalan que el videojuego les sirve para motivar ejercicios de motricidad fina con el teclado y el ratón a la vez que consiguen recompensas al jugar.

Otro de los aspectos que han considerado que se trabajan en el aula es el conteo; siendo destacado por el 1\%. Justifican su decisión por los diferentes contadores presentes en el juego y son utilizados en muchos casos para resolver cámaras o calcular elementos necesarios para poder solventar algún obstáculo. El 2\% restante señala que no ven una clara conexión del videojuego a aspectos matemáticos que pudieran tratarse en la asignatura.

\section{DISCUSIÓN Y CONCLUSIONES}

Esta investigación pretendía analizar la inclusión del videojuego Portal 2 como recurso de interés en el aula de Educación Infantil. Se puede señalar que los datos recabados en este estudio exploratorio arrojan una interesante fotografía de las consideraciones existentes sobre los videojuegos por parte de las futuras maestras y maestros de Educación Infantil. Se ha podido observar que, a pesar de las numerosas investigaciones y literatura existentes sobre los beneficios y la viabilidad educativa de los videojuegos, éstos siguen adoleciendo de una percepción negativa lastrada por la existencia de prejuicios como la generación de adicción, agresividad y las temáticas violentas. Se puede señalar que su consideración meramente lúdica es tenida en cuenta por una parte importante de los individuos analizados. No obstante, atendiendo al objetivo planteado para esta investigación, conocer la percepción del alumnado del Grado de Educación Infantil de la Facultad de Educación de la Universidad de Cádiz sobre la utilización del videojuego Portal 2 para trabajar nociones matemáticas sobre la orientación y la percepción espacial, el alumnado mantiene que la aplicación de este videojuego es propicia con una mayoría significativa. No obstante, destacan que su inclusión en este nivel educativo debe ser como recurso complementario junto con otros materiales como la pizarra digital, el libro de texto, entre otros. También, ubican la utilización de los videojuegos como recurso en Educación Primaria y Secundaria por encima de Educación Infantil. Sin embargo, al contextualizar los aprendizajes que pueden tratarse con Portal 2, lo vinculan con el área de conocimiento del entorno; focalizando sobre los aprendizajes relacionados con el ámbito de las matemáticas los individuos investigados consideran que la percepción viso-espacial se trabaja de modo efectivo con la utilización de este videojuego (Green, Bavelier, 2006). Además de la implementación de diferentes estrategias relacionadas con la lógica y la autonomía personal orientada a la resolución de problemas. El estudio sigue la línea de otros anteriores (Shute, Ventura y Ke, 2015; Vaddi, Toups, Dolgov, Wehbe y Nacke, 2016) que ya manifestaron la utilidad de Portal 2 en la adquisición de habilidades cognitivas y no cognitivas. 
Tras los resultados obtenidos se pretende realizar un estudio más amplio que ayude a delimitar el grado de adquisición de aprendizajes realizados con el videojuego para conocer de un modo más preciso como afecta el proceso de juego a los aprendizajes que pudieran obtenerse con su uso. Finalmente, cabe señalar que los discentes deben aprovechar la coyuntura tecnológica actual con el fin de renovar recursos y extraer todos los elementos beneficiosos para un aprendizaje motivador y eficaz.

\section{REFERENCIAS BIBLIOGRÁFICAS}

Alsina, A. (2011). Relaciones y cambios de posición y forma. Educación Matemática en contexto: de 3 a 6 años. (103-123). Barcelona: Horsori.

Green, C. S., \& Bavelier, D. (2006). Effect of action video games on the spatial distribution of visuospatial attention. Journal of experimental psychology: Human perception and performance, 32(6), 1465

Gros, B., \& Bernat, A. (2008). Videojuegos y aprendizaje. Barcelona: Graó.

Guerra, J. (2017). Estudio evaluativo de prevención del acoso escolar con un videojuego. Universidad de Extremadura, Cáceres.

López, I. (2014). ¿Qué es un videojuego?: claves para entender el mayor fenómeno cultural del siglo XXI. Sevilla: Arcade.

McGonigal, J. (2011). Reality is broken: why games make us better and how they can change the world. New York: Penguin Group.

Prensky, M. (2001). Digital game-based learning. New York: McGraw-Hill.

Revuelta, F. I., \& Guerra, J. (2012). ¿Qué aprendo con videojuegos? Una perspectiva de meta-aprendizaje del videojugador. Revista de Educación a Distancia, (33).

Shute, V. J., Ventura, M., \& Ke, F. (2015). The power of play: The effects of Portal 2 and Lumosity on cognitive and noncognitive skills. Computers \& education, 80, 58-67.

Vaddi, D., Toups, Z., Dolgov, I., Wehbe, R. R., \& Nacke, L. (2016). Investigating the Impact of Cooperative Communication Mechanics on Player Performance in Portal 2. 
